Research Paper

\title{
Microstructure Evolution in Metal Nanostructures under Extreme Conditions of Temperature and Strain Rate
}

\author{
V GUPTA $^{1, *}$, N M GHONIEM ${ }^{1}, \mathrm{R}_{\text {CRUM }}^{2}, \mathrm{G} \mathrm{PO}^{2}, \mathrm{D} \mathrm{SEIF}^{2}$, S V PRIKHODKO*, H A COLORADO ${ }^{2}$, \\ B RAMIREZ ${ }^{2}$ and C GÁMEZ ${ }^{2}$ \\ ${ }^{1}$ Department of Materials Science and Engineering, UCLA \\ ${ }^{2}$ Department of Mechanical and Aerospace Engineering, UCLA
}

(Received on 29 February 2016; Accepted on 10 March 2016)

\begin{abstract}
This paper explores a fundamental connection between ductility and domain size in metallic solids under extreme conditions of cryogenic temperatures and strain rates $\left(10^{8} \mathrm{~s}^{-1}\right)$. A series of novel experiments, backed by multiscale modelling and transmission electron microscopy (TEM) analysis, are presented that involve loading of TEM-ready single crystal nanopillar samples of $\mathrm{Cu}$ of varying lengths $(50 \mathrm{~nm}$ to $1 \mathrm{~mm})$ and aspect ratios $(50 \mathrm{~nm}$ to $100 \mathrm{~nm}$ in diameter) by laser-generated stress waves of sub-nanosecond rise times, under extreme conditions of strain rate $\left(>10^{8} \mathrm{~s}^{-1}\right)$ and temperature $(100 \mathrm{~K})$. The nucleation stress for Shockley partials, which can be taken as a proxy for the onset of ductile deformation, was measured to be only $1 \mathrm{GPa}$. This is an order of magnitude lower than the previously measured values of 35 GPain bulk geometries. TEM observations show remarkable ability of the material to re-arrange itself through motion of dislocations to form subgrain boundaries within a very short duration of only few nanoseconds.
\end{abstract}

Keywords: Ductility; Shock Loading; FCC Metals; TEM; MD Simulations

\section{Introduction}

In this paper we explore the fundamental connection between small size and ductility in metallic solids and explore if such a relationship can be maintained even under ultrahigh strain rate loading and cryogenic temperatures. Most engineering materials behave in a brittle fashion under such conditions. This even includes the Face Centred Cubic (FCC) metals such as copper that are among the most ductile materials when deformed at room temperature and lower loading rates. On a fundamental level, this brittleness under extreme conditions results from the very low mobility of nucleated and pre-existing dislocations as the phonon drag mechanism that controls their mobility in FCC metals at room temperature and lower loading rates becomes inefficient because of the increase in the rate-dependent viscous drag force on the dislocations. Body Centred Cubic (BCC) metals such as Tungsten (W) which are already brittle at room temperature tend to become even more brittle at extreme conditions. This room temperature brittleness in $\mathrm{W}$ is related to the necessity of nucleating kink pairs on the non-planar core structure of screw dislocations by overcoming of the Peierlsenergy barrier. This process is energetically more demanding than the phonon drag mechanism in FCC metals which does not require overcoming the Peierls barrier. Because of the latter, FCC metals are ductile at room temperature. Regardless, both FCC and BCC metals deform in a brittle fashion under extreme conditions. A question that arises naturally is can the microstructures of these metals be designed such that they respond in a ductile fashion under all loading conditions? This query has motivated our research because of its significance to our society as this would allow design of energy-absorbing blast resistant structures and armours that are critically needed for defence and space exploration applications. In addition, the possibility of making $\mathrm{W}$ ductile at room temperature is very tantalizing as it would expand its application domain considerably and also lead to

*Author for Correspondence: E-mail:vgupta@ucla.edu; Tel.: (310) 8250223 
tremendous cost savings as because of its brittleness $\mathrm{W}$ must be heated during most machining operations.

Two very encouraging results from our on-going research show the possibility of accomplishing the above goal by limiting the size of the deforming microstructural domains to within several hundred nanometres. First, our Discrete Dislocation Dynamics (DDD) simulations of deforming submicron single crystal tungsten pillars show that the role of screw dislocations in BCC metals quickly diminishes with decreasing pillar diameter at all temperatures and strain rates. Specifically, the results (Cui et al., 2016) indicate that in small domain size, $\mathrm{W}$ deforms by fundamentally altering the motion of screw dislocations. They too move via the phonon drag mechanism at room temperature without the need for nucleating the kink pairs. This would then indicate that in small domains $\mathrm{W}$ could be ductile at room temperature and higher loading rates. Compression and bending experiments on submicron size $\mathrm{W}$ pillars at different temperatures are being conducted to confirm these results.

The second result was from our experimental study that showed that $\mathrm{Cu}$ nano-pillars in the $200 \mathrm{~nm}$ to $500 \mathrm{~nm}$ diameter range deformed in a ductile fashion even under extreme strain rate $\left(\sim 10^{8} \mathrm{~s}^{-1}\right)$ and temperature (100K-200K) conditions. The nucleation stress for Shockley partials was measured to be $\sim 1$ GPa which is an order of magnitude lower than its previous well-established value in bulk. These results suggest that there may be a fundamental connection between small size and ductility that cannot be perturbed either by ultrahigh strain rate loading or cryogenic temperatures.

In this paper we present resultsfrom our FCC copper study in which TEM-ready single crystal nanopillar samples of $\mathrm{Cu}$ and $\mathrm{Al}$ of varying lengths $(50 \mathrm{~nm}$ to $1 \mathrm{~mm}$ ) and aspect ratios $(50 \mathrm{~nm}$ to $100 \mathrm{~nm}$ in diameter) were loaded by laser-generated stress waves of sub-nanosecond rise times, under extreme conditions of strain rate $\left(>10^{8} \mathrm{~s}^{-1}\right)$, and temperature (100K). Since shock loading is performed in TEMready samples it provides an extremely powerful tool to experimentally correlate the evolved structures to experimental parameters of pressure, strain rate, and temperature with minimal modelling. Additionally, multi-scale modelling simulations are carried out virtually in the same geometries as the TEM and shock experiments. This allows for a direct comparison between experiments and simulations to establish the scientific underpinning for the experimentally observed new deformation mechanisms.

\section{Sample Preparation}

A Focussed Ion Beam (FIB)-based procedure was used to make $\mathrm{Cu}$ nano-pillars of widely ranging geometries $(100 \mathrm{~nm}-1500 \mathrm{~nm}$ tall and $50-500 \mathrm{~nm}$ in diameter) starting from a $\mathrm{Cu}(001)$ substrate plate (Fig. 1a,d). Four groups of 6 identical pillars, each spanning a $6 \mathrm{~mm}$ dia area, were prepared in a Nova 600 Dual Beam focused-ion-beam/scanning electron microscope (FIB/SEM) system using gallium ions at $0.5 \mathrm{nA}$ current and an accelerating voltage of $30 \mathrm{kV}$. This level of ion beam current ensured no damage due to Ga implantation such as those reported at 10$20 \mathrm{nA}$ where $\mathrm{Ga}$ content of $20 \%$ at up to a depth of $35 \mathrm{~nm}$ was measured. This can result in dislocation pile-ups and therefore make the separation of the damage caused by the shock wave difficult.Selected area electron diffraction (SAED) pattern of the reference pillar in Fig. 1d confirms the pillar to be single crystal albeit it is populated with pre-existing dislocations with density $7.9 \times 10^{14} \mathrm{~m}^{-2}$ as shown in the bright-field TEM image (Fig. 1a) of the reference unshocked pillar when viewed along the [001] zone axis.

For bending experiments (Colorado et al., 2013), nano-pillars were fabricated directly on the TEM grids purchased from OmniprobeAutoProbe ${ }^{\mathrm{TM}}$. Asreceived grids were annealed for 20 hours at $150^{\circ} \mathrm{C}$ to remove residual stresses. Nano-pillars were then fabricated at the tip of the Omniprobe grid post using the FIB process discussed above. Three pillars were made on each post, with a centre-to-centre separation of about $5 \mu \mathrm{m}$. All pillars were approximately $1 \mu \mathrm{m}$ in length and $100 \mathrm{~nm}$ in diameter. To further protect the pillars during sample handling and transfer between TEM and SEM stages and shock apparatus, the thickness of the Omniprobe grid post was kept significantly higher $(40 \mathrm{~mm})$ than that of the pillars.

\section{Laser Spallation Test Facility for Shock Loading}

A Laser Spallation Facility, developed previously to measure the tensile strengths of interfaces (Yuan and Gupta, 1993) was adapted to load the pillars in 

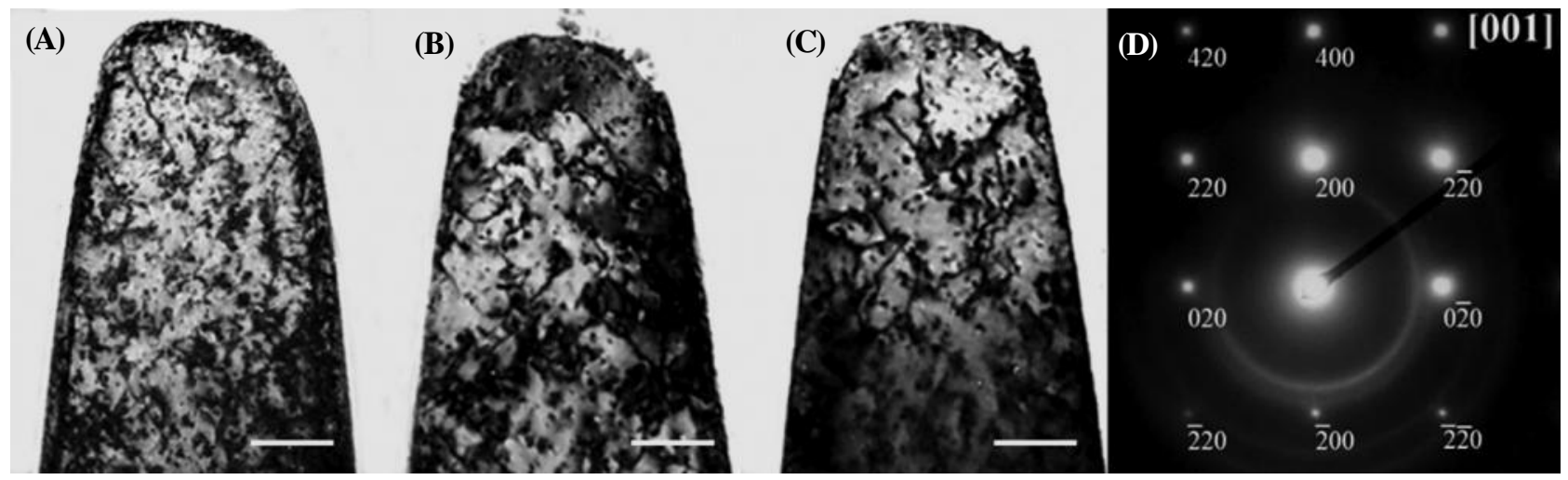

Fig. 1: TEM images of three different $\mathrm{Cu}(001)$ pillars acquired (A) as-prepared and after shock-loading using laser fluence of (B) $39.5 \mathrm{~kJ} / \mathrm{m}^{2}$ and (C) $93.4 \mathrm{~kJ} / \mathrm{m}^{2}$. The scale bar in all images is $100 \mathrm{~nm}$ and (D) Selected area electron diffraction (SAED) pattern of the pillar shown in (A)

compression (Fig. 2) and bending (Fig. 3) by lasergenerated stress waves of $1 \mathrm{~ns}$ rise time and $16-20 \mathrm{~ns}$ total duration. In both experiments, the $\mathrm{Cu}$ sample was sandwiched between a 1-mm-thick glass substrate and a 9-mm-thick brass plate as shown in Fig. 2. The back surface of the glass substrate was coated with a $0.5-\mu \mathrm{m}$-thick, laser absorbing $\mathrm{Al}$ film, which was constrained from the top by a transparent $20-50 \mu \mathrm{m}$ thick layer of waterglass. The stress waves were generated inside the glass slide by exfoliating the constrained $\mathrm{Al}$ film by focusing a nominal $8 \mathrm{~ns}$ long Nd:YAG laser pulse over a $2.22 \mathrm{~mm}$ diameter area of the $\mathrm{Al}$ film. The axes of the pillars were aligned parallel to the wave propagation direction for compression testing and perpendicular for bending experiments.

A new experimental apparatus was developed to shock load nanostructures, in-situ, at cryogenic temperatures. This is a fairly sophisticated setup comprising of an environmental cell capable of maintaining pre-cooled nano-pillars at cryogenic temperatures while still able to receive the shock wave generated by the laser.

The stress state inside the copper pillars was determined using a two-step procedure. In the first step, the incident stress wave $\sigma(\mathrm{t})$ generated inside the glass plate was measured in a separate experiment using interferometry. In this experiment, an isolated glass plate was shock-loaded under the same stress wave generation conditions (laser fluence and thicknesses of glass substrate, $\mathrm{Al}$ film, and waterglass layers) as those used for shock loading of the $\mathrm{Cu}$ pillars. The free surface velocity $v(\mathrm{t})$, recorded using the optical interferometer, is used to calculate $\sigma(\mathrm{t})$ by equation:

$$
\sigma(\mathrm{t})=0.5 \rho c v(t+h / c)-v(t-h / c)
$$

where $c(=2289 \mathrm{~m} / \mathrm{s})$ is the longitudinal wave speed in the glass, and $\rho$ and $h$ areits density, and thickness, respectively (Pronin and Gupta, 1997). In step 2, the exact stress-state inside each pillar was determined using a finite element (FE) simulation with $\sigma(t)$ as an input. The FE model was implemented in ABAQUS. The mesh size was kept uniform in all domains so as to prevent the spurious wave reflection effects that are known to occur at material interfaces and furthermore the time step of $0.2 \mathrm{~ns}$ was used to satisfy the numerical stability requirement relating $\Delta t$ to element size $\Delta x$ through the wave velocity c : $\Delta t$ $<\Delta x / c$. The local stresses from obtained from the FE model were used for carrying out theMolecular Dynamics (MD) simulations under shock compression (Youssef et al., 2013).

\section{TEM and SEM Analysis}

After shock-loading, the pillars were lifted off the substrate using FIB and mounted on TEM grids. Electron-transparent $(<100 \mathrm{~nm})$ cross-sectional samples were prepared by FIB milling of the pillars at a low accelerating voltage, $5 \mathrm{kV}$. TEM, scanning TEM (STEM), SAED, along with energy dispersive $\mathrm{X}$-ray spectroscopy (EDS) measurements were carried out using a JEM-100CX (JEOL) TEM operated at $100 \mathrm{kV}$ and a Titan (FEI) S/TEM operated at $300 \mathrm{kV}$. Fig. 1 shows TEM images of pre- and post-shocked $\mathrm{Cu}$ nano-pillars. These were analysed 


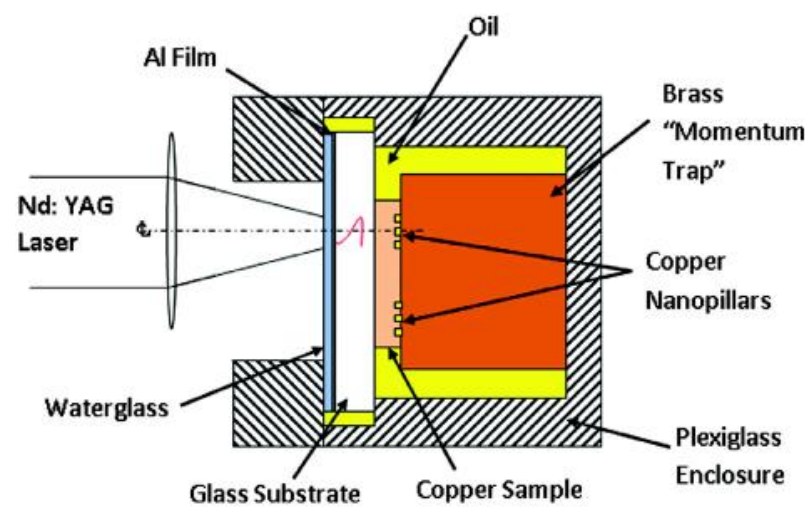

Fig. 2: Shock compression setup

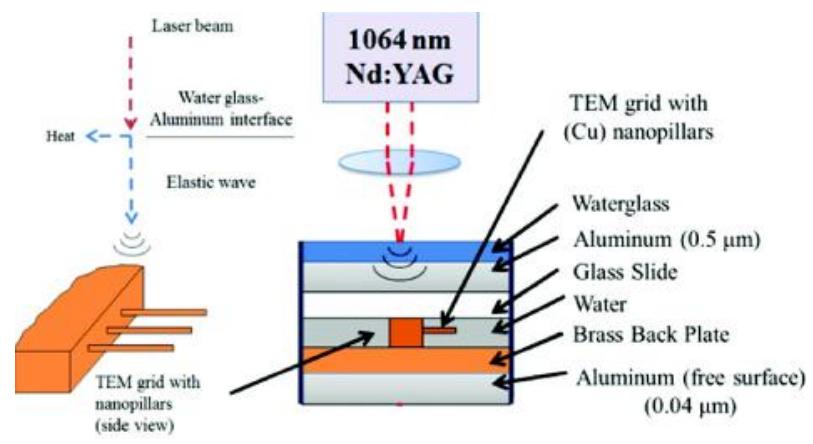

Fig. 3: Shock bending setup

to measure the dislocation density changes and infer the nucleation stress for the Shockley partials by using interferometric measurements and FEM analysis as discussed above. This process yielded a nominal compressive stress of only $1 \mathrm{GPa}$ corresponding to the nucleation of Shockley partials! This is very low compared to the $35 \mathrm{GPa}$ estimated for their nucleation and propagation in 2-D nano-film geometry by our MD simulationsand similar other works in the High Energy Materials field. This is a very surprising finding. Since such events are a proxy to the onset of ductile behaviour one must conclude that the extremely small size of pillars leads to this behaviour. This was further confirmed by our MD simulations, discussed in the next section.

A further support for the above thesis was provided by the results obtained on copper pillars that were precooled to $100 \mathrm{~K}$ before shock loading in the new setup. Remarkably, the pillars deformed by accommodating a very large compressive strain either through substantial axial shortening (Fig. 4C) or
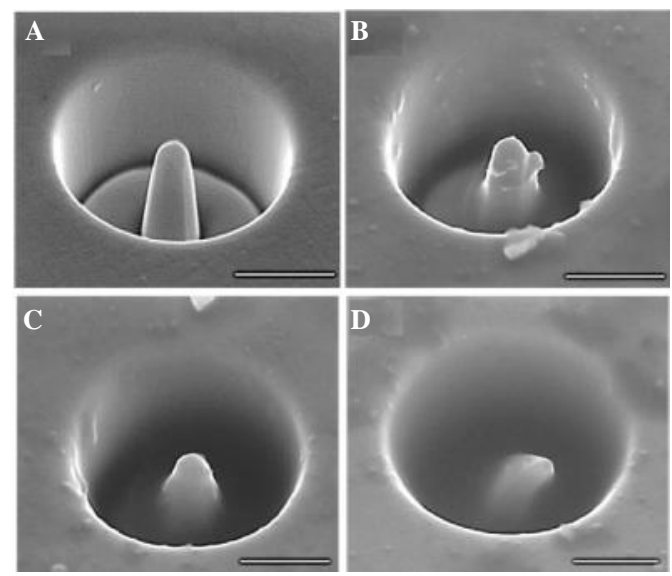

Fig. 4: Shocked Cu pillars. Scale: $1 \mu \mathrm{m}$

through the formation of a heavily displaced shear band (Fig. 4B). When the pillars were bent by orienting their axes $90^{\circ}$ to the wave propagation direction (shock bending), pillars were found to bend by $180^{\circ}$ without fracture even at such $\left(10^{8} \mathrm{~s}^{-1}\right)$ ultrahigh strain rates (Colorado et al., 2013). This is evidenced in Fig. 5 which displays SEM images of a pre-shocked pillar that was progressively bent at increasing stress wave amplitudes, Figs. 5B and 5C.

Figure 6 shows typical TEM images and SAED patterns acquired from the most bent $\mathrm{Cu}$ pillar before $(\mathrm{A}, \mathrm{B})$ and after $(\mathrm{C}, \mathrm{D})$ it was shocked with a stress wave generated with $450 \mathrm{~mJ}$ laser pulse. The TEM image shows straight line contrast suggestive of a twin grain sub-boundary within the crystal, which was confirmed by indexing of SAED pattern. A few dislocations are also visible in the image. From the SAED pattern, we have identified this to be a twin boundary separating two (111) crystals. Dark-field image of the pillar obtained using (200) reflection is shown as an inset in Fig. 6A. The brighter contrast in the image highlights one of the twin crystals, referred to here as the parent crystal. After the shock treatment, we notice the presence of the twin crystal in the tip of the pillar (Fig. 6C). However, the twin boundary visible in Fig. 6A before laser treatment appears to have changed shape. The SAED pattern in Fig. 6D and the dark-field image reveal a change in the microstructure of the pillar after shock treatment. The contrast visible in the dark-field image is due to the presence of a large number of dislocations in the pillar. SAED pattern in Fig. 6D is indicative of multiple subgrain boundaries and development of a 


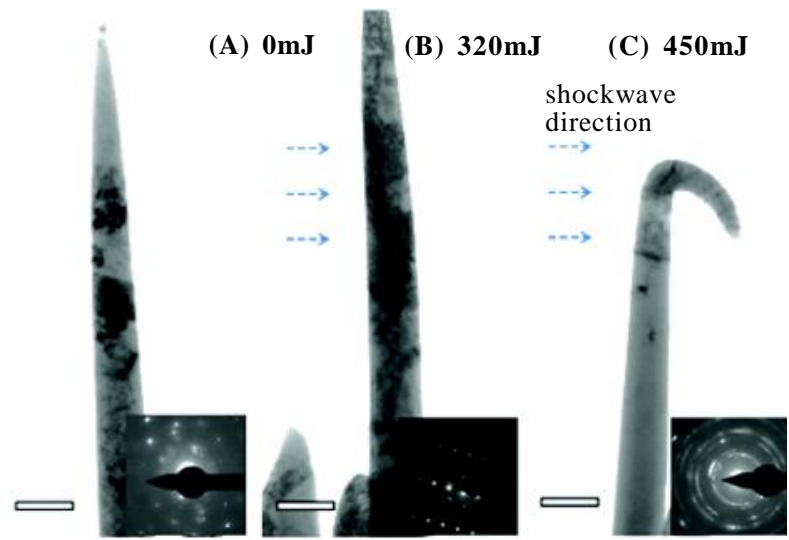

Fig. 5: TEM bright-field images of the nanopillar before (A) and after laser shot at $320 \mathrm{~mJ}(B)$ and $450 \mathrm{~mJ}$ (C). Scale bars in all images are $200 \mathrm{~nm}$

texture at the nanoscale.

High resolution TEM (HRTEM) image of the post shocked pillar shown in Figure $5 \mathrm{C}$ in the vicinity of a twin boundary (boxed region in Fig. 7C) is shown in the central panel of Fig. 7. Fourier transforms (FT) of the three boxed regions of the HRTEM image that are shown in three separate panels on the very right in Fig. 7 clearly show the presence of new grains. This is a remarkable finding as such a material rearrangement has occurred within few nanoseconds.

\section{Simulations}
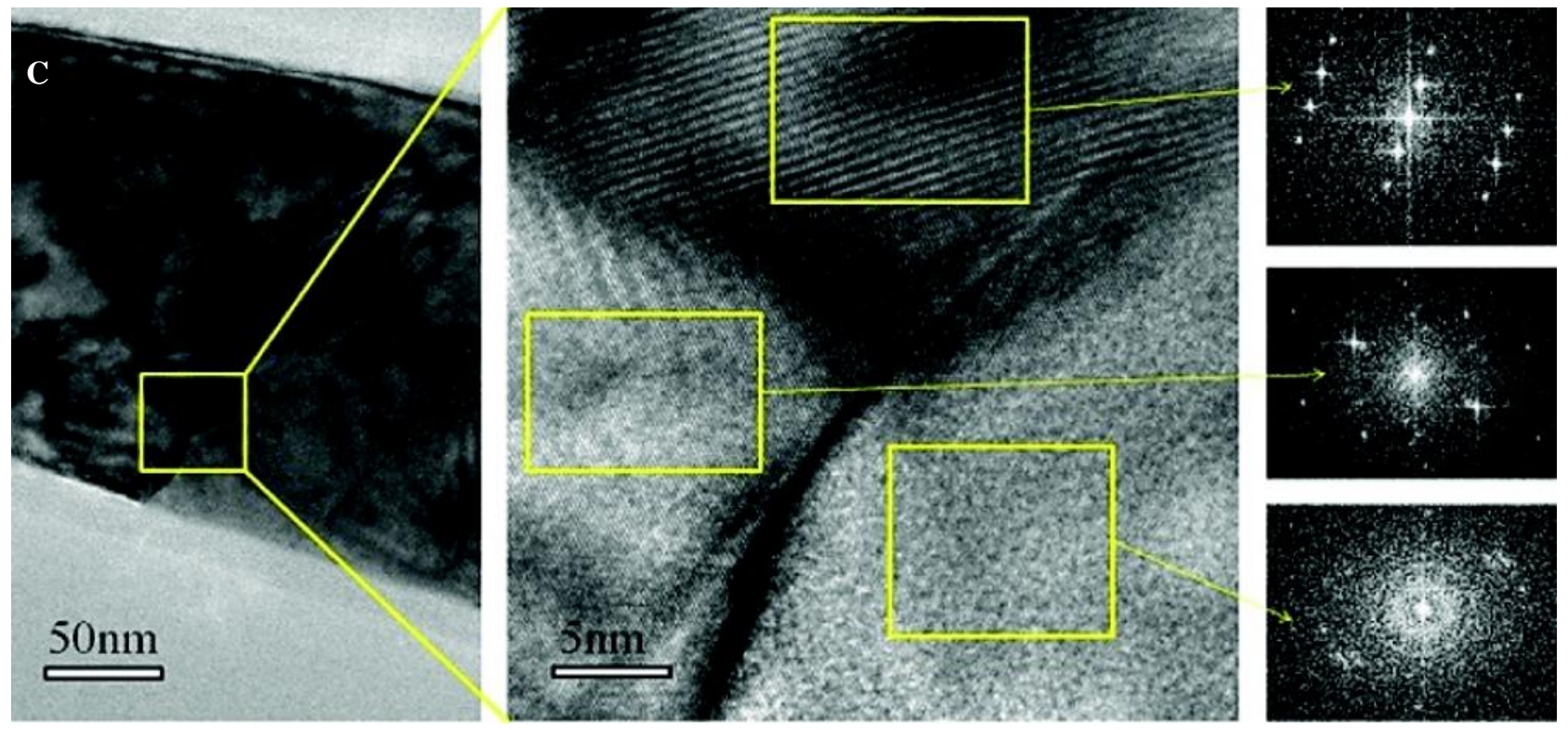

Fig. 7: HRTEM images of the area of the pillar boxed in Figure 7 (c). Panel to the right shows FFT patterns of different areas boxed in the central panel. Two areas taken above the twin boundary has different orientation 


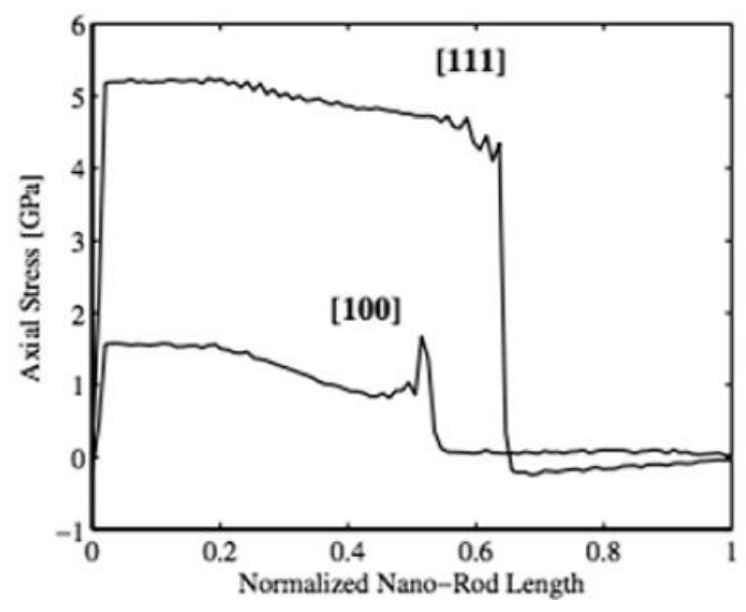

Fig. 8: Elastic wave profiles of two $80 \mathrm{~nm}$ diameter, [100]and [111]-oriented copper nano-rods, shocked loaded by a rigid piston traveling at $100 \mathrm{~m} / \mathrm{s}$ and $200 \mathrm{~m} / \mathrm{s}$, respectively

roughly $25 \times 25 \times 101 \mathrm{~nm}^{3}$ ( 5.5 x $10^{6}$ atoms), with periodic and free boundaries on the faces transverse and normal to the loading direction, respectively. Thus, the transverse periodicity likens the computational cell to a nano-film. Nano-rods, on the other hand, were cylindrical having free boundaries in all directions. While a range of sizes were examined for the nanorods giving similar results, we present here results for nano-rods with diameters of $\sim 54 \mathrm{~nm}$ and lengths of $\sim 81 \mathrm{~nm}\left(\sim 14.1 \times 10^{6}\right.$ atoms $)$.

All simulations began with a ramped thermal equilibration scheme using an isothermal-isobaric (NPT) ensemble, bringing the atoms from 0 to 300 $\mathrm{K}$ over 50 ps. Once this temperature was achieved, amicrocanonical (NVE) ensemble was then used and the shock loading is applied. To do this, we designated the first few atomic planes normal to the loading direction as a rigid piston. To produce the shock loading, atoms belonging to the piston were given a prescribed constant velocity into the material for all time. Typical shock simulation times were approximately $30 \mathrm{ps}$, with time steps of $1 \mathrm{fs}$. Two different crystal orientations were simulated to analyse the effects of orientation. In the first, the loading direction ( $\mathrm{z}$ ) was the [100] direction with $\mathrm{x}$ and $\mathrm{y}$ being the [010] and [001] directions, respectively. In the second, the loading direction was the [111] with $\mathrm{x}$ and y being the $\left[\begin{array}{ll}\overline{1} & 10\end{array}\right]$ and $\left[\begin{array}{ll}11 & \overline{2}\end{array}\right]$ directions, respectively.

Shock loading simulations of nano-films with lateral periodic boundary conditions showed that impact stresses greater than $35 \mathrm{GPa}$ are required to nucleate and propagate dislocations in the sample. While consistent with earlier MD simulations (Remington et al., 2006; Bringa et al., 2006) these pressures are significantly greater than the pressures generated in our laser experiments on nanopillars $(\sim 1$ GPa) where plasticity-induced surface activation was observed. To explain this discrepancy the response of nano-pillars was modelled next. Following impact, we found the nano-pillar to undergo two temporal regimes: (1) fully elastic and (2) plastic release. Initially, as the pillar is impacted, an elastic compression wave is generated and begins its travel through the crystal. Depending on the impact strength, for some period of time we observe full elasticity prior to the nucleation of dislocations. This delay occurs as the free surfaces allow for volume relaxations as a means of impact energy absorption. Thus, in the fully elastic regime, the elastic wave propagates through the crystal and bulging of the pillar occurs near the piston interface. Once the bulging reaches a maximum for the material the second regime of plastic release initiates. This occurs through the nucleation of dislocations near the piston interface. Unlike the case of nano-films, however, this nucleation does not occur at the shock front and does not occur homogeneously in the pillar's cross-section. With the maximum radial displacements occurring at the surface due to volumetric bulging, we find Shockley partial dislocations to nucleate preferentially from the surface, at the piston interface. Impact velocities and stresses required for the onset of plasticity and subsequent melting are much lower in nano-pillars as compared to the bulk nano-films. When shocked with impact velocities on the order of those used in the nano-film cases, the nano-pillars instantaneously melt as the piston passes through the crystal. For the [100] oriented rod, we have found that reducing the impact velocity to $\sim 0.1 \mathrm{~km} / \mathrm{s}$, nucleation of Shockley partials just begins to commence. This corresponds to an axial stress of $\sim 1.0-1.5 \mathrm{GPa}$, which is fully consistent with the stress level measured for triggering such eventsin our laser shock experiments.

Fig. 8 shows the wave profile in the pillar just when dislocation nucleation begins to occur. Note that the purely elastic wave front is already more than halfway through the length of the rod by the time the plastic release occurs at the piston interface. Fig. 9 

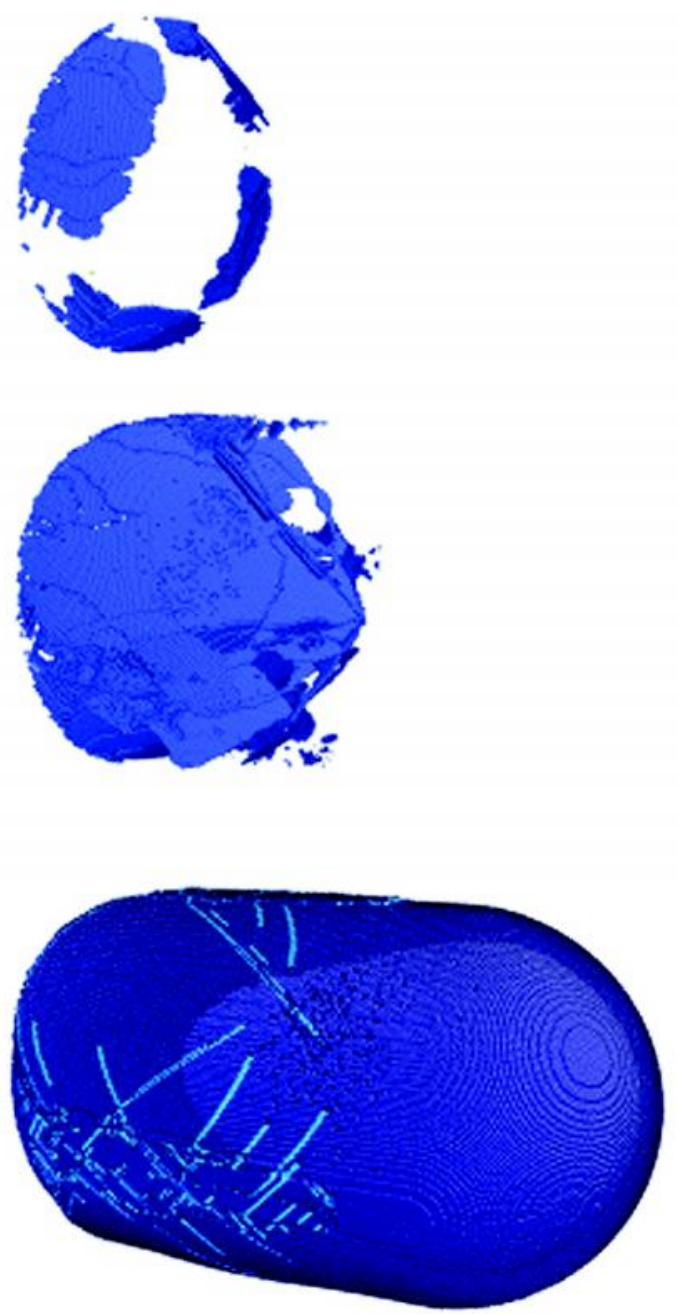

Fig. 9: Shock compression of $80 \mathrm{~nm}$ diameter [100] $\mathrm{Cu}$ pillar at $5 \mathrm{GPa}$. The top image is at $4.8 \mathrm{ps}$, the middle is at $12.8 \mathrm{ps}$, and the bottom image shows the final state. The nucleation of dislocations initiates at the free surfaces at much lower stresses, as compared the simulations of the bulk nano-films

shows the results of the impact of a [100] oriented nanopillar by a rigid piston traveling at $0.25 \mathrm{~km} / \mathrm{s}$

Materials Research at the National Science Foundation under Grant Number 1024353.

\section{References}

Bringa E M, Rosolankova K, Rudd R E, Remington B A, Wark J

S, Duchaineau M, Kalantar D H, Hawreliak J and Belak J (2006) Shock deformation of face-centered-cubic metals
( $5 \mathrm{GPa})$. The images show the dislocation nucleation and evolution phases as the shock wave proceeds, and the resulting surface damage. We note in these images the dominance of partial dislocations with stacking faults of very large surface area. Slip, primarily by expansion of stacking faults, continues to take place until Shockley partials reach opposing surfaces, leaving permanent damage at the free surfaces in the form of atomic steps. Experimentally, such regions of surface damage can act as effective zones for chemical activity, which were observed in our experiments (Youssef et al., 2013). In contrast to the nano-film simulations, here slip and surface step creation continue to dissipate the wave energy until the plastic wave motion ceases and the crystal is permanently deformed.

\section{Conclusions}

The experimental results on FCC copper presented here establish that in small domains the dislocation nucleation stress is significantly reduced which is a proxy for the onset of ductility. Experiments also show ductile failure mechanisms at extreme conditions of ultrahigh strain rate loading and low temperature. The MD simulations support these experimental observations.

Present work is focused on performing shock experiments on submicron $\mathrm{W}$ pillars to confirm the DDD simulation results and establish the relationship between small size and ductility for all metals. Thus, we remain very optimistic about establishing this thesis which ultimately will guide development of small grain size polycrystals of BCC and FCC metals that will retain both strength and ductility under all strain rates and temperature conditions.

\section{Acknowledgement}

This research was supported by the Division of

on subnanosecond timescales Nature Materials 5 805-809

Cui Y, Po G and Ghoniem N M (2016) Temperature insensitivity of the flow stress in body-centered cubic micropillar crystals Acta Materialia 108 128-137

Colorado H A, Navarro A, Prikhodko S V, Yang J M, Ghoniem N and Gupta V (2013) Ultrahigh strain-rate bending of copper 
nanopillars with laser-generated shock waves Journal of Applied Physics 114 233-510

Pronin A and Gupta V (1993) Interferometry on diffuse surfaces in high-velocity measurements Review of Scientific Instruments 64 2233-2236

Remington B A, Allen P, Bringa E M, Hawreliak J, Ho D, Lorenz K T, Lorenzana H, McNaney J M, Meyers M A, Pollaine S W, Rosolankova K, Sadik B, Schneider M S, Swift D, Wark J and Yaakobi B (2006) Material dynamics under extreme conditions of pressure and strain rate Materials Science and Technology 22 474-488
Seif D, Po G, Crum R, Gupta V and Ghoniem N M (2014) Shockinduced plasticity and the Hugoniot elastic limit in copper nano films and rods Journal of Applied Physics 115054301

Youssef G, Crum R, Prikhodko S V, Seif D, Po G, Ghoniem N and Gupta V (2013) The influence of laser-induced nanosecond rise-time stress waves on the microstructure and surface chemical activity of single crystal Cu nanopillars Journal of Applied Physics 113084309

Yuan J and Gupta V (1993) Measurement of interface strength by the modified laser spallation technique I. Experiment and simulation of the spallation process Journal of Applied Physics 74 2388-2396. 